\title{
Tendencias en el uso del cuerpo en el valle del Atuel (sur de Mendoza) durante el Holoceno tardío final
}

\author{
Eva A. Peralta \\ Recibido 18 de febrero 2020. Aceptado 27 de julio 2020
}

\section{RESUMEN}

En este trabajo se propone explorar los marcadores de uso del cuerpo para conocer el estilo de vida de los grupos humanos del valle del Atuel (sur de Mendoza) durante los últimos 2000 años, en el marco de la interacción caza-recolección/producción de alimentos. Específicamente se busca evaluar las demandas mecánicas, así como discutir posibles asociaciones con la movilidad y con las actividades físicas vinculadas a la subsistencia, para lo cual se estudia la variación de los cambios entésicos y de las lesiones articulares. Se comparan las tendencias entre unidades espaciales (desiertos de Monte y Patagonia) y por bloques temporales (2000-1000 años AP y 1000-200 años AP). Los resultados indican que las muestras de ambos desiertos exhibieron patrones diferenciados que pueden interpretarse como el resultado de estrategias particulares asociadas al uso del cuerpo. Para el primer bloque temporal, en Monte la movilidad podría ser menor que en Patagonia y los miembros superiores estarían menos exigidos, mientras que no se observan cambios sustanciales en el segundo bloque. Estas tendencias podrían también explicarse por los efectos del sexo y la edad, o del tamaño corporal de los individuos, variables hasta el momento desconocidas, dadas las características de las muestras disponibles.

Palabras clave: Transición caza-recolección/producción de alimentos; Movilidad; Cambios entésicos; Lesiones articulares.

\section{ABSTRACT}

TRENDS IN BODY USE DURING THE LATER LATE HOLOCENE IN THE ATUEL RIVER VALLEY, SOUTH OF MENDOZA. This paper explores body use markers to evaluate the lifestyle of human groups in the Atuel River Valley, south of Mendoza, during the past 2000 years using the framework of hunter-gatherer/ food production interaction. Specifically, the aim is to evaluate the mechanical demands associated with mobility and physical activities linked to subsistence. To this end, the variation in entheseal changes and indicators of joint damage were studied. Trends between spatial units (Monte and Patagonia deserts) and temporal blocks (2000-1000 BP and 1000-200 BP) were also compared. The study results show that samples from both deserts exhibit differentiated patterns that could be related to differences in body use. For the first temporal block, mobility in Monte was lower than in Patagonia and the upper limbs were less stressed. For the second time block, there are no substantial changes. Trends could be explained by sex, age and body size effects. Currently, the latter variables are unknown due to sample limitations.

Keywords: Hunting-gathering/food production transition; Mobility; Entheseal changes; Joint injuries.

\section{INTRODUCCIÓN}

Desde fines de 1990, en el sur de Mendoza, el significado de la presencia de cultígenos (e.g., Zea mays) se estudió desde distintas líneas de evidencia para evaluar su rol en la vida de las poblaciones humanas (Gil, 2003; Gil et al., 2006; Neme y Gil, 2012). El consumo de recursos vegetales

Eva A. Peralta. Consejo Nacional de Investigaciones Científicas y Técnicas (CONICET). Instituto de Evolución, Ecología Histórica y Ambiente (IDEVEA). Av. Gral. J. J. Urquiza 314 (CP 5600), San Rafael, Mendoza, Argentina. E-mail: evaailenperalta@gmail.com

Intersecciones en Antropología 21(2), julio-diciembre: 187-200. 2020. ISSN-e 1850-373X

https://doi.org/10.37176/iea.21.2.2020.510

Facultad de Ciencias Sociales - UNICEN - Argentina 
domesticados y el posible desarrollo de actividades productivas se explicaron como el resultado de una amplitud en la dieta en el marco de un proceso de intensificación en la explotación de los recursos hacia 2000 años AP, cuya génesis se encuentra en un desbalance entre la demografía humana y la disponibilidad de los recursos explotados (Neme, 2007; Neme y Gil, 2012).

Aunque se ha registrado y explicado un consumo variable de recursos vegetales domesticados a partir de los 2000 años AP, aún se desconoce si fueron producidos localmente y cuál fue su impacto en las estrategias organizativas de los grupos humanos (Gil et al., 2017; Peralta, 2019). Asimismo, es escasa la información acerca de la variabilidad que tuvieron estos procesos en relación con las diferencias ecológicas que presentan los ambientes del sur de Mendoza, puntualmente los desiertos de Monte y Patagonia. En un trabajo previo se realizó una aproximación inicial al estudio de los patrones de uso del cuerpo en un contexto de interacción entre la caza-recolección y la producción de alimentos (Peralta, 2017). En una muestra más pequeña, se articularon análisis de cambios entésicos y lesiones articulares con información isotópica para discutir alteraciones en la movilidad y problematizar las interpretaciones basadas en los extremos caza-recolección/agricultura. Se contrastaron tendencias en miembros superiores con respecto a los inferiores, y no se detectaron modificaciones en las estrategias de movilidad vinculadas con la implementación de actividades productivas.

En este contexto, se propone explorar los marcadores de uso del cuerpo para evaluar el estilo de vida de los grupos humanos del valle del Atuel (sur de Mendoza) durante los últimos 2000 años, en el marco de la interacción caza-recolección/producción de alimentos. En esta oportunidad, con una muestra más amplia que permite comparaciones a nivel espacial y temporal, se busca incrementar los resultados previamente obtenidos y mejorar la discusión sobre el uso del cuerpo en un área de interacción entre dos modos de subsistencia. Para esto se estudiará la variación de los cambios entésicos (CE) y de las lesiones articulares (LA), indicadores que han sido ampliamente usados en el campo de la bioarqueología para la reconstrucción de patrones de actividad física y estilos de vida del pasado. Uno de los tópicos más abordados desde los CE y las LA corresponde a los cambios en los sistemas de subsistencia, aunque no se definieron tendencias claras: para algunos autores, disminuyen los niveles de actividad física con la introducción de la producción de alimentos, mientras que para otros aumentan (Larsen, 1984; Rooselvelt, 1984; Bridges, 1989, 1992, 1994; Molleson, 1994; Eshed et al., 2004; Marchi et al., 2006; Larsen y Ruff, 2011).

Los cambios entésicos son modificaciones del tejido óseo que se producen al nivel de las inserciones musculares o entesis (Benjamin et al., 1986; Benjamin y Ralphs, 1998; Benjamin et al., 2002; Jurmain y Villotte, 2010). Este fenómeno tiene lugar debido a que el organismo humano busca un equilibrio entre la estructura ósea y la masa muscular asociada, teniendo en cuenta que tanto los huesos como los músculos son tejidos dinámicos y activos que se modifican continuamente (Wolff, 1892). Bajo condiciones sostenidas de estrés mecánico, el hueso refuerza las áreas donde se inserta el músculo para disipar la fuerza y la tensión que se ejercen sobre él (Wolff, 1892). Las lesiones articulares refieren a los cambios degenerativos a nivel de las articulaciones, con una alta prevalencia en el registro bioarqueológico (Rogers y Waldron, 1995; Manuila et al., 2000; Berenbaum, 2013, Waldron, 2009). Afectan al cartílago y generan, en sus últimas etapas, modificaciones en la superficie ósea asociada, las cuales implican la destrucción (porosidad y eburnación) y la producción de hueso (osteofitos) (Neumann, 1999; Solomon, 2001; Rogers et al., 2004; Lagier, 2006; Seibel et al., 2006).

Se han planteado una serie de limitaciones que alertan a la hora de realizar inferencias sobre actividad física a partir de CE y LA (Santos et al., 2011; Jurmain et al., 2012; Henderson et al., 2013; Villotte y Knüsel, 2013). La de mayor relevancia se relaciona con la etiología multifactorial de estos marcadores, ya que en su desarrollo no solo interviene la actividad física, sino que también influyen la edad, el sexo, las patologías e incluso las condiciones genéticas (Jurmain, 1977, 1980; Stirland, 1998; Seibel et al., 2006; Henderson, 2008; Nolte y Wilczak, 2013; Villotte y Knüsel, 2013; Henderson et al., 2017). Teniendo estos factores en consideración y controlándolos en la medida de lo posible, los CE y las LA constituyen una línea de evidencia factible en estudios de carácter poblacional, con preguntas concretas acerca de modos de vida hipotéticamente contrastantes, como lo propuesto al inicio del trabajo.

\section{Hipótesis y expectativas}

El estado de las investigaciones en el sur de Mendoza indica un vector N-S para la dispersión de la agricultura y una mayor ubicuidad, tanto del 
registro de macrorrestos de cultígenos, como de la señal isotópica del maíz en sitios localizados en el desierto de Monte en comparación con el desierto de Patagonia (Gil et al., 2006; Gil y Neme, 2010; Gil et al., 2017). Tomando esto como base, la hipótesis principal de este trabajo indica que durante el Holoceno tardío final (2000-200 años AP), las poblaciones humanas del desierto de Monte y del desierto de Patagonia tuvieron subsistencias diferentes: mientras que en Monte se incorporó la producción de alimentos, en Patagonia predominó la caza-recolección. Asimismo, se propone la continuidad a lo largo del tiempo del modelo agricultor en Monte, mientras que en Patagonia se mantiene el modo de vida cazador-recolector.

Como hipótesis derivada se proponen patrones diferenciados de uso del cuerpo que responden al tipo de subsistencia, lo cual se refleja en CE y LA específicos en cada uno de los desiertos. En algunos contextos se ha detectado una tendencia a la disminución del estrés mecánico en miembros inferiores como consecuencia de una reducción en la movilidad (Holt, 2003; Holt y Formicola, 2008; Larsen y Ruff, 2011; Marchi et al., 2011; Stock et al., 2011; Salega, 2016; Sparacello et al., 2020). Asimismo, los miembros superiores reflejarían en mayor medida actividades manuales vinculadas con la subsistencia (Eshed et al., 2004; Henderson, 2008; Villotte et al., 2010; Schrader, 2012; Henderson et al., 2018; Varalli et al., 2020).
De acuerdo con esta información se modelaron expectativas para miembros inferiores y superiores de Monte y Patagonia. Se espera que los miembros inferiores de los individuos de Monte presenten una prevalencia menor de CE y LA respecto de las tendencias identificadas en los individuos de Patagonia para la misma porción anatómica, debido a las diferencias en la carga física relacionada con la movilidad. Asimismo, se esperan mayores prevalencias de CE y LA en los miembros superiores de los individuos de Monte con respecto a los individuos de Patagonia, como respuesta a las mayores demandas físicas asociadas con la diversidad de actividades en un contexto de producción de alimentos (labrado de la tierra, cosecha, molienda). También se espera que estas tendencias para cada desierto se mantengan a lo largo del tiempo.

\section{MATERIALES Y MÉTODOS}

La muestra analizada en este trabajo corresponde a enterratorios localizados en el valle del Atuel (Figura 1, Tabla 1). Se incluyeron en el análisis todas las muestras disponibles dentro de esta área. Estas corresponden a las colecciones osteológicas del Museo de Historia Natural de San Rafael. Se realizó una primera instancia de selección determinada por la presencia de elementos óseos analizables. Se destaca que los elementos del esqueleto $-70^{\circ} 30^{\prime} 0^{\prime \prime}$

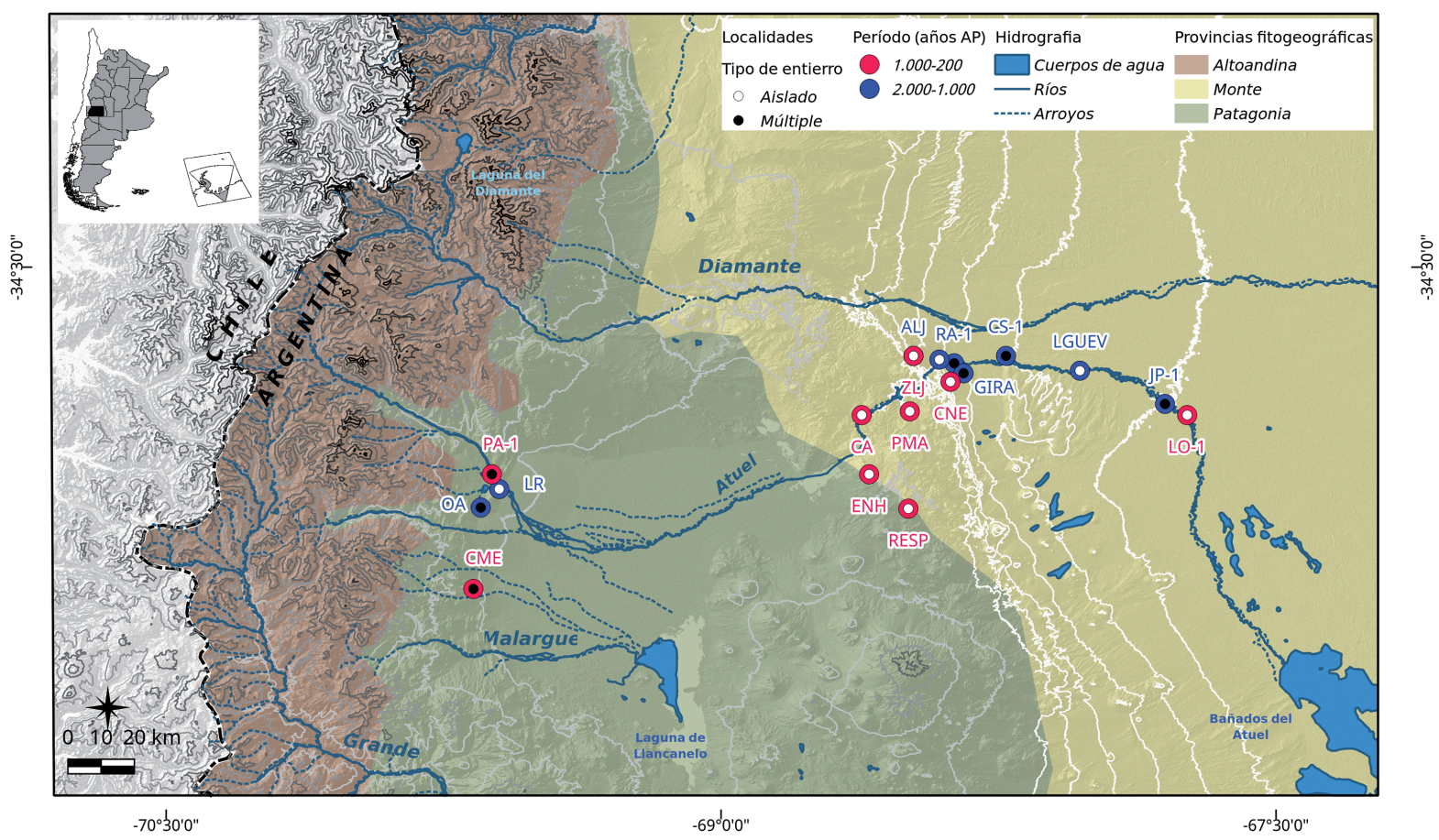

Figura 1. Mapa con la localización de los entierros analizados en este trabajo. 


\begin{tabular}{|c|c|c|c|c|c|c|c|c|c|c|c|c|}
\hline 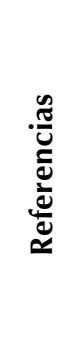 & 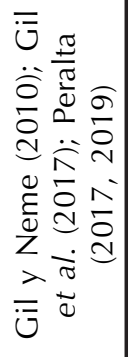 & 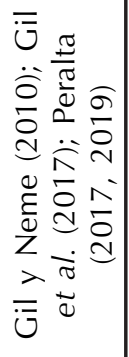 & 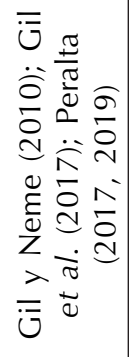 & 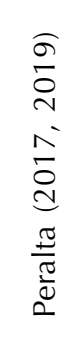 & 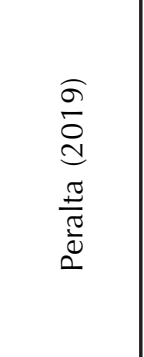 & 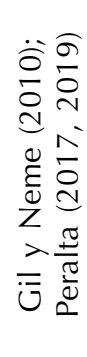 & 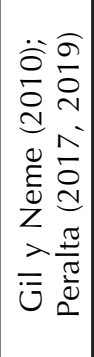 & 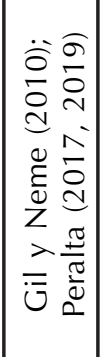 & 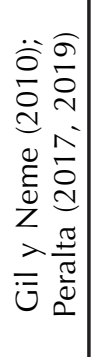 & 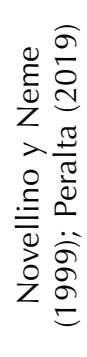 & 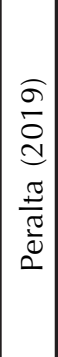 & $\begin{array}{l}\sigma \\
\sigma \\
\frac{d}{d} \\
\frac{\pi}{\pi} \\
\frac{\pi}{0} \\
2\end{array}$ \\
\hline 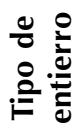 & $\sum_{\Psi}$ & $\sum_{\Psi}$ & $\sum_{U}$ & 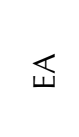 & $\sum_{u}$ & $\mathbb{w}$ & 『 & $\mathbb{w}$ & 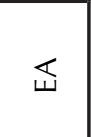 & $\sum_{u}$ & $\sum_{\Psi}$ & $\overleftarrow{w}$ \\
\hline$\dot{\bar{g}}$ & 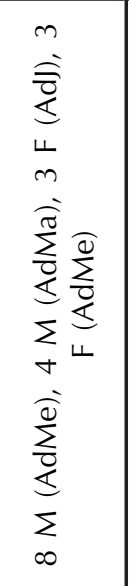 & 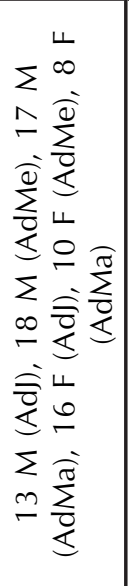 & 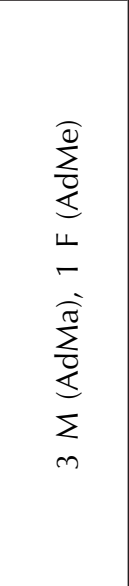 & $\begin{array}{l}\frac{0}{0} \\
\frac{0}{0} \\
\Sigma\end{array}$ & 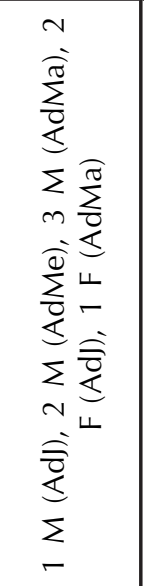 & $\begin{array}{l}\overline{\bar{\alpha}} \\
\bar{\Sigma}\end{array}$ & $\begin{array}{l}\frac{0}{0} \\
\frac{\sum_{0}}{2} \\
\Sigma\end{array}$ & $\begin{array}{l}\overline{\overline{0}} \\
\bar{\Sigma}\end{array}$ & $\begin{array}{l}\hat{\overline{0}} \\
\bar{\Sigma}\end{array}$ & $\begin{array}{l}\frac{0}{0} \\
\frac{0}{0} \\
\sum \\
\vdots \\
0\end{array}$ & 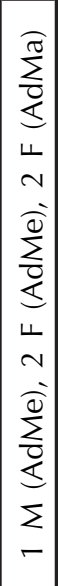 & $\begin{array}{l}\frac{\pi}{2} \\
\frac{\pi}{2} \\
\Sigma \\
\Sigma\end{array}$ \\
\hline 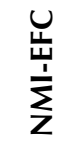 & $\stackrel{m}{=}$ & $\hat{\sim}$ & $\sim$ & - & $\stackrel{N}{\simeq}$ & - & - & - & - & - & $\nabla$ & - \\
\hline 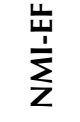 & $\stackrel{\bullet}{\circ}$ & $\dot{m}$ & $m$ & - & $\simeq$ & - & - & - & - & - & $\nabla$ & - \\
\hline$\frac{\overleftarrow{c}}{\sum_{\mathbf{n}}^{\mathbf{n}}}$ & $\stackrel{\Omega}{\sigma}$ & $\tilde{m}$ & $\sim$ & - & $\sigma$ & - & - & - & - & - & Ln & - \\
\hline 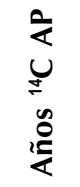 & 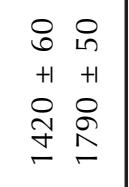 & 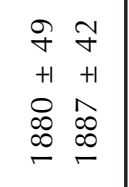 & 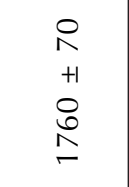 & $\begin{array}{l}\circ \\
m \\
+1 \\
\vdots \\
\Xi \\
\end{array}$ & 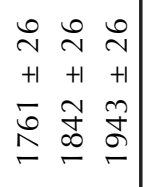 & \begin{tabular}{l}
$\stackrel{2}{2}$ \\
+1 \\
\multirow{+}{+}{}
\end{tabular} & $\begin{array}{l}\text { Ln } \\
m \\
+1 \\
\infty \\
\infty \\
\llcorner\end{array}$ & $\begin{array}{l}\infty \\
\sim \\
+1 \\
0 \\
+ \\
\infty\end{array}$ & 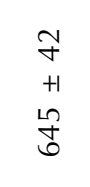 & $\begin{array}{l}\circ \\
\stackrel{0}{+1} \\
8 \\
\stackrel{2}{2}\end{array}$ & $\begin{array}{c}n \\
m \\
+1 \\
\bar{z} \\
m\end{array}$ & $\begin{array}{l}\stackrel{\llcorner}{N} \\
+1 \\
\infty \\
\stackrel{2}{\sigma}\end{array}$ \\
\hline 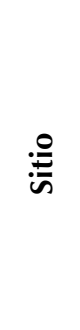 & 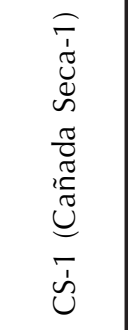 & 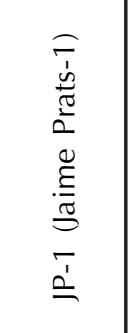 & 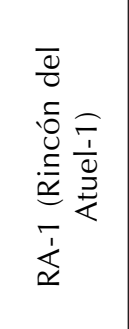 & 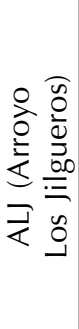 & 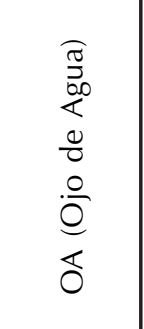 & 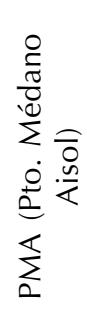 & 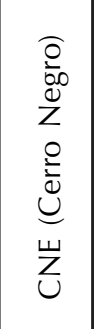 & 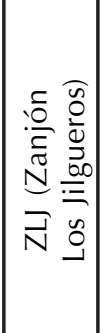 & $\begin{array}{l}\frac{\sigma}{\bar{\sigma}} \\
\stackrel{\sigma}{=} \\
0 \\
0\end{array}$ & 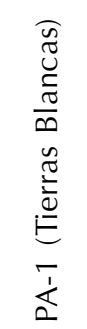 & 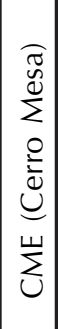 & 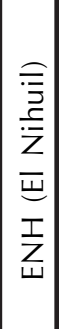 \\
\hline$\frac{\mathscr{\Xi}}{\infty}$ & \multicolumn{4}{|c|}{$\begin{array}{l}\bar{Z} \\
\stackrel{O}{z}\end{array}$} & $\underset{\Sigma}{\Sigma}$ & \multicolumn{4}{|c|}{$\begin{array}{l}\text { Ỹ } \\
\text { Ȯ }\end{array}$} & \\
\hline
\end{tabular}

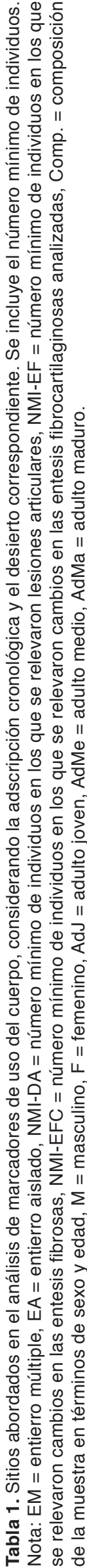


apendicular se encontraron, en líneas generales, mejor conservados y representados que los del esqueleto axial. Algunos individuos que componen la muestra se caracterizan por provenir de inhumaciones aisladas de carácter primario, mientras que la mayor parte de los elementos analizados proviene de entierros múltiples mezclados. Estos últimos se excavaron varias décadas atrás y se han caracterizado como osarios en los que se ha perdido la asociación por individuo (Peralta, 2019). En estos contextos solo fue posible obtener información acerca del sexo y de la edad para una parte de los elementos analizados. En la Tabla 1 se presenta la composición sexo-etaria disponible para los entierros múltiples y aislados, estimada a partir de rasgos morfológicos de coxales y/o cráneos (véase Peralta, 2019 para los detalles sobre la estimación del NMI y medidas de abundancia anatómica para cada uno de los sitios considerados en este trabajo). Teniendo en cuenta los objetivos primarios de esta investigación, se priorizó un análisis de escala amplia, de acuerdo con el cual fueron comparadas grandes unidades espacio-temporales. Esta elección se realizó bajo el supuesto de que las diferencias en el patrón de subsistencia serían suficientes entre las unidades comparadas como para observar patrones distintivos en el uso del cuerpo, independientemente del sexo y edad del individuo.

La muestra analizada alcanzó un número mínimo estimado de 80 individuos y se organizó considerando la localización fitogeográfica de los sitios (Monte o Patagonia), así como su temporalidad de acuerdo con dos bloques temporales: uno entre 2000-1000 años AP y otro entre 1000-200 años AP. Este recorte temporal responde a la ausencia de restos óseos humanos con cronologías previas a los 2000 años. Asimismo, dada la necesidad de explorar los cambios acontecidos luego de la aparición de los cultígenos en el registro arqueológico (ca. 2000 años AP), se estableció operativamente una segmentación de la muestra por la cual se agruparon los sitios cada 1000 años, siguiendo la tendencia general de los fechados radiocarbónicos, en la que una parte de los sitios presentan fechados próximos a los 2000 años y otra parte luego de los 1000 años AP (Tabla 1). Se conformaron cuatro conjuntos: MON1 (muestras de monte fechadas entre 2000-1000 años AP, NMI = 57), PAT1 (muestras de Patagonia fechadas entre 2000-1000 años AP, NMI = 12), MON 2 (muestras de monte fechadas entre 1000-200 años AP, NMI = 4) y PAT2 (muestras de Patagonia fechadas entre 1000200 años $\mathrm{AP}, \mathrm{NMI}=7$ ) (Tabla 1). En el análisis se consideraron solo los elementos correspondientes a individuos adultos (con las líneas de fusión epifisiaria cerradas) que no presentaran señales patológicas de origen infeccioso o metabólico. En la Tabla S1 se detallan las articulaciones y entesis relevadas y el $n$ correspondiente en cada caso.

Para el relevamiento de las LA se siguió la propuesta desarrollada por Luna y colaboradores (2017), que considera el relevamiento tanto de la intensidad como del porcentaje de afectación y distingue las manifestaciones en el borde articular de las que se producen en el interior de la superficie. En este sentido, los autores destacan que las manifestaciones en el borde, en forma de proliferaciones óseas, se asocian con situaciones crónicas de estrés mecánico, mientras que al interior de la superficie se produce porosidad derivada de procesos inflamatorios con una etiología diversa, generalmente de índole infecciosa o congénita (Rothschild, 1997; Aufderheide y RodríguezMartín, 1998; Manuila et al., 2000; Rogers et al., 2004; Lagier, 2006; Waldron, 2009; Luna et al., 2017). Considerando este marco conceptual, la formación de hueso nuevo en los márgenes de la articulación (o también labiaciones, osteofitos o lipping) es concebida en este trabajo como el rasgo más relevante al momento de evaluar el desarrollo de las LA. Asimismo, no se evalúa solamente la ocurrencia de las proliferaciones, sino que se consideran también su longitud y extensión. Si bien para algunos autores las manifestaciones de LA requieren la coocurrencia de varios rasgos para hablar de condiciones patológicas, en este caso seleccionamos una metodología que permitiera registrar y comparar cambios sutiles vinculados con situaciones reiteradas de estrés mecánico. De acuerdo con la propuesta metodológica seguida, cada articulación fue dividida en cuatro cuadrantes, a cado uno de los cuales le fue asignado un grado de manifestación entre 0 y 3 , manteniendo siempre la separación entre borde y superficie interior (Tabla S2). Posteriormente se dividió la sumatoria de los grados observados por los cuadrantes efectivamente relevados. Finalmente, estos porcentajes se clasificaron en una escala de 0 a $3(0=$ no afectado, $1=$ menos de $25 \%, 2=$ entre 25 y $50 \%, 3$ = más de $50 \%$ ). Los datos obtenidos fueron agrupados posteriormente teniendo en cuenta, por un lado, cada grupo articular (hombro, codo, muñeca, cadera, rodilla y tobillo) y, por otro, el sector del esqueleto (miembros superiores vs. miembros inferiores). 
Para el relevamiento de entesis de tipo fibrocartilaginosas se empleó el "método Coimbra" desarrollado por Henderson et al. (2016a y 2016b). De acuerdo con este, se dividió la entesis en dos zonas (zona 1 y zona 2) y se registraron una serie de rasgos: en zona 1, formación ósea (FO1) y erosión (ERO1); en zona 2, cambio textural (CT), formación ósea (FO), erosión (ERO), porosidad fina (PF), macroporosidad (MPO) y cavidades (CAV). A cada rasgo se le asignó un grado de manifestación entre 0 y 2, excepto para $\mathrm{CT}$, para el que se registró presencia (1) o ausencia (0) (Tabla S3). Dado que el método Coimbra no se aplica al estudio de entesis fibrosas, para el relevamiento de este tipo de entesis se utilizó la metodología propuesta por Hawkey y Merbs (1995). Esta elección se realizó considerando que es el método más comúnmente usado tanto en nuestra región como en áreas aledañas, lo que posibilitaría estudios comparativos (Scabuzzo, 2010, 2012; Giannotti, 2016; Salega, 2016). Según estos autores, en cada entesis se registran tres rasgos: robusticidad (ROB), lesiones de estrés (LE) y osificaciones (OSI). Se asignó a cada rasgo un grado de manifestación entre 0 y 3 (Tabla S4). Para el análisis se realizaron comparaciones por entesis $y$, en una segunda instancia, agrupando los datos por sector del esqueleto (miembros superiores vs. miembros inferiores).

En el procesamiento de los resultados relacionados con las entesis fibrocartilaginosas se empleó el análisis de componentes principales. Para el testeo de diferencias significativas entre los conjuntos se empleó el test de la $U$ de Mann-Whitney, que se ajusta al análisis de variables ordinales en muestras que no presentan una distribución normal. Se tomó como límite de significancia un valor $p$ de 0,05. Todos los análisis se realizaron empleando PAST 3.15 (Hammer et al., 2001).

\section{RESULTADOS}

\section{Patrones de lesiones articulares}

El análisis por articulación mostró que, para todos los conjuntos comparados (MON1, PAT1, MON2, PAT2), las articulaciones con mayor presencia de cambios asociados a lesión articular fueron el codo, la rodilla y el tobillo (Tablas 2 y 3). Si bien la prevalencia de LA en el codo fue más alta en PAT1 en comparación con MON1, en este último conjunto se observaron grados moderados a severos de manifestación y cambios a nivel de superficie. La rodilla presentó una prevalencia levemente mayor de LA en MON1 a nivel de borde en comparación con PAT1, aunque este último conjunto incluye grados severos de manifestación en el borde y prevalencias más altas a nivel de la superficie. El tobillo se vio afectado solo en el borde, tanto en MON1 como en PAT1; se observan prevalencias más altas en PAT1, aunque en grados más leves. No se detectaron diferencias significativas entre las articulaciones de MON1 y PAT1 ni a nivel del borde ni de la superficie. En la muestra de PAT2, el codo, la rodilla y el tobillo mostraron mayores prevalencias de LA en comparación con la muestra de MON2, tanto en borde como en superficie. Se observaron diferencias significativas en el codo y la muñeca, a nivel del borde, y en la rodilla a nivel del borde y la superficie.

Al comparar los porcentajes de afección en miembros superiores e inferiores para cada desierto y período cronológico, se observaron mayores prevalencias de lesiones articulares en miembros inferiores de MON1 en relación con los de PAT1, y mayores prevalencias en los miembros superiores de PAT1 con respecto a MON1 (Tabla 4). Los miembros superiores e inferiores de PAT2 se mostraron más afectados con respecto a los de MON2. Solo en el periodo 1000-200 años AP se corroboraron diferencias significativas (Tabla 4).

\section{Cambios entésicos}

\section{Entesis fibrosas}

En MON1, el pronador cuadrado y el supinador fueron las entesis fibrosas que mostraron un porcentaje más alto de robusticidad, incluyendo grados moderados (Tabla S5). Las lesiones de estrés y osificaciones solo se presentaron en el pectoral mayor y el braquiorradial, en un bajo porcentaje y en grados leves. En los miembros inferiores, la tuberosidad glútea y la línea soleal fueron las entesis que mostraron las prevalencias más altas de robusticidad, lesiones de estrés y osificaciones, incluyendo grados moderados a severos de manifestación. En PAT1, el pectoral mayor fue la entesis, que exhibió el porcentaje más alto de robusticidad, mientras que el pronador cuadrado y el supinador presentaron los porcentajes más bajos. No se registraron lesiones ni osificaciones en las entesis de miembros superiores. En los miembros inferiores, los porcentajes de robusticidad fueron más altos en las entesis de los cuádriceps y la línea soleal. No se 
Tendencias en el uso del cuerpo en el valle del Atuel (sur de Mendoza) durante el Holoceno... Intersecciones en Antropología 21(2), julio-diciembre. 2020. ISSN-e 1850-373X

\begin{tabular}{|c|c|c|c|c|c|c|c|c|}
\hline Conjunto & Grado & $n / \%$ & Hombro & Codo & Muñeca & Cadera & Rodilla & Tobillo \\
\hline \multirow{8}{*}{$\begin{array}{l}\text { MON1/ } \\
\text { BORDE }\end{array}$} & \multirow{2}{*}{0} & $\mathrm{n}$ & 27 & 113 & 76 & 46 & 39 & 28 \\
\hline & & $\%$ & 77,1 & 65,3 & 76,8 & 97,9 & 62,9 & 62,2 \\
\hline & \multirow{2}{*}{1} & $\mathrm{n}$ & 7 & 50 & 21 & 1 & 22 & 16 \\
\hline & & $\%$ & 20 & 28,9 & 21,2 & 2,1 & 35,5 & 35,6 \\
\hline & \multirow{2}{*}{2} & $\mathrm{n}$ & 1 & 7 & 2 & 0 & 1 & 1 \\
\hline & & $\%$ & 2,9 & 4 & 2 & 0 & 1,6 & 2,2 \\
\hline & \multirow{2}{*}{3} & $\mathrm{n}$ & 0 & 3 & 0 & 0 & 0 & 0 \\
\hline & & $\%$ & 0 & 1,7 & 0 & 0 & 0 & 0 \\
\hline \multirow{8}{*}{$\begin{array}{c}\text { MON1/ } \\
\text { SUPERFICIE }\end{array}$} & \multirow{2}{*}{0} & $\mathrm{n}$ & 30 & 166 & 99 & 45 & 59 & 45 \\
\hline & & $\%$ & 88,2 & 88,8 & 96,1 & 95,7 & 95,2 & 100 \\
\hline & \multirow{2}{*}{1} & $\mathrm{n}$ & 4 & 18 & 3 & 2 & 3 & 0 \\
\hline & & $\%$ & 11,8 & 9,6 & 2,9 & 4,3 & 4,8 & 0 \\
\hline & \multirow{2}{*}{2} & $\mathrm{n}$ & 0 & 2 & 1 & 0 & 0 & 0 \\
\hline & & $\%$ & 0 & 1,1 & 1 & 0 & 0 & 0 \\
\hline & \multirow{2}{*}{3} & $\mathrm{n}$ & 0 & 1 & 0 & 0 & 0 & 0 \\
\hline & & $\%$ & 0 & 0,5 & 0 & 0 & 0 & 0 \\
\hline \multirow{8}{*}{$\begin{array}{l}\text { PAT1/ } \\
\text { BORDE }\end{array}$} & \multirow{2}{*}{0} & $\mathrm{n}$ & 5 & 12 & 6 & 11 & 11 & 4 \\
\hline & & $\%$ & 100 & 54,5 & 75 & 100 & 68,8 & 57,1 \\
\hline & \multirow{2}{*}{1} & $\mathrm{n}$ & 0 & 10 & 2 & 0 & 4 & 3 \\
\hline & & $\%$ & 0 & 45,5 & 25 & 0 & 25 & 42,9 \\
\hline & \multirow{2}{*}{2} & $\mathrm{n}$ & 0 & 0 & 0 & 0 & 0 & 0 \\
\hline & & $\%$ & 0 & 0 & 0 & 0 & 0 & 0 \\
\hline & \multirow{2}{*}{3} & $\mathrm{n}$ & 0 & 0 & 0 & 0 & 1 & 0 \\
\hline & & $\%$ & 0 & 0 & 0 & 0 & 6,3 & 0 \\
\hline \multirow{8}{*}{$\begin{array}{c}\text { PAT1/ } \\
\text { SUPERFICIE }\end{array}$} & \multirow{2}{*}{0} & $\mathrm{n}$ & 5 & 22 & 8 & 11 & 14 & 7 \\
\hline & & $\%$ & 100 & 100 & 100 & 100 & 82,4 & 100 \\
\hline & \multirow{2}{*}{1} & $\mathrm{n}$ & 0 & 0 & 0 & 0 & 3 & 0 \\
\hline & & $\%$ & 0 & 0 & 0 & 0 & 17,6 & 0 \\
\hline & \multirow{2}{*}{2} & $\mathrm{n}$ & 0 & 0 & 0 & 0 & 0 & 0 \\
\hline & & $\%$ & 0 & 0 & 0 & 0 & 0 & 0 \\
\hline & \multirow{2}{*}{3} & $\mathrm{n}$ & 0 & 0 & 0 & 0 & 0 & 0 \\
\hline & & $\%$ & 0 & 0 & 0 & 0 & 0 & 0 \\
\hline \multicolumn{3}{|c|}{$\begin{array}{c}\text { Valores p (MON1 vs. } \\
\text { PAT1, borde) }\end{array}$} & 0,25115 & 0,46128 & 0,94218 & 0,66008 & 0,77865 & 0,84948 \\
\hline \multicolumn{3}{|c|}{$\begin{array}{c}\text { Valores p (MON1 vs. } \\
\text { PAT1, superficie) }\end{array}$} & 0,44773 & 0,09923 & 0,58409 & 0,50998 & 0,08154 & - \\
\hline
\end{tabular}

Tabla 2. Prevalencias ( $n$ y \%) de lesiones articulares en borde y superficie en MON1 y PAT1, considerando grados de severidad. Se incluyen valores $p$ del test Mann-Whitney.

registraron lesiones de estrés, y las osificaciones se hicieron presentes solo en la línea áspera y en los cuádriceps. Diferencias significativas entre desiertos solo se registraron en el pectoral mayor, en cuanto a robusticidad $(p=0,01672)$.

En MON2, los cambios en las entesis fibrosas mostraron porcentajes más bajos con respecto a la muestra de PAT2. En MON2, el pectoral mayor, el pronador cuadrado y el supinador fueron las entesis más afectadas por la robusticidad en los miembros superiores, y se registraron solo en

\section{Entesis fibrocartilaginosas}

Dado el mayor número de rasgos registrados por cada entesis, se realizó un análisis de componentes principales que permitió ponderar estadísticamente las variables y observar las de mayor peso en la diferenciación de las muestras. En la comparación de los miembros superiores del primer bloque temporal (MON1-PAT1), el Componente 1 explicó el 42,9\% de la varianza, siendo el cambio textural (CT) la 


\begin{tabular}{|c|c|c|c|c|c|c|c|c|}
\hline Conjunto & Grado & $n / \%$ & Hombro & Codo & Muñeca & Cadera & Rodilla & Tobillo \\
\hline \multirow{8}{*}{$\begin{array}{l}\text { MON2/ } \\
\text { BORDE }\end{array}$} & \multirow{2}{*}{0} & $\mathrm{n}$ & - & 13 & 9 & 4 & 8 & 4 \\
\hline & & $\%$ & - & 81,3 & 100 & 100 & 88,9 & 80 \\
\hline & \multirow{2}{*}{1} & $\mathrm{n}$ & - & 3 & 0 & 0 & 1 & 1 \\
\hline & & $\%$ & - & 18,8 & 0 & 0 & 11,1 & 20 \\
\hline & \multirow{2}{*}{2} & $\mathrm{n}$ & - & 0 & 0 & 0 & 0 & 0 \\
\hline & & $\%$ & - & 0 & 0 & 0 & 0 & 0 \\
\hline & \multirow{2}{*}{3} & $\mathrm{n}$ & - & 0 & 0 & 0 & 0 & 0 \\
\hline & & $\%$ & - & 0 & 0 & 0 & 0 & 0 \\
\hline \multirow{8}{*}{$\begin{array}{c}\text { MON2/ } \\
\text { SUPERFICIE }\end{array}$} & \multirow{2}{*}{0} & $\mathrm{n}$ & - & 15 & 9 & 4 & 9 & 5 \\
\hline & & $\%$ & - & 93,8 & 100 & 100 & 100 & 100 \\
\hline & \multirow{2}{*}{1} & $\mathrm{n}$ & - & 1 & 0 & 0 & 0 & 0 \\
\hline & & $\%$ & - & 6,3 & 0 & 0 & 0 & 0 \\
\hline & \multirow{2}{*}{2} & $\mathrm{n}$ & - & 0 & 0 & 0 & 0 & 0 \\
\hline & & $\%$ & - & 0 & 0 & 0 & 0 & 0 \\
\hline & \multirow{2}{*}{3} & $\mathrm{n}$ & - & 0 & 0 & 0 & 0 & 0 \\
\hline & & $\%$ & - & 0 & 0 & 0 & 0 & 0 \\
\hline \multirow{8}{*}{$\begin{array}{l}\text { PAT2/ } \\
\text { BORDE }\end{array}$} & \multirow{2}{*}{0} & $\mathrm{n}$ & 5 & 10 & 9 & 7 & 4 & 3 \\
\hline & & $\%$ & 50 & 34,5 & 52,9 & 77,8 & 21,1 & 33,3 \\
\hline & \multirow{2}{*}{1} & $\mathrm{n}$ & 4 & 13 & 6 & 2 & 7 & 6 \\
\hline & & $\%$ & 40 & 44,8 & 35,3 & 22,2 & 36,8 & 66,7 \\
\hline & \multirow{2}{*}{2} & $\mathrm{n}$ & 1 & 4 & 2 & 0 & 6 & 0 \\
\hline & & $\%$ & 10 & 13,8 & 11,8 & 0 & 31,6 & 0 \\
\hline & \multirow{2}{*}{3} & $\mathrm{n}$ & 0 & 2 & 0 & 0 & 2 & 0 \\
\hline & & $\%$ & 0 & 6,9 & 0 & 0 & 10,5 & 0 \\
\hline \multirow{8}{*}{$\begin{array}{c}\text { PAT2/ } \\
\text { SUPERFICIE }\end{array}$} & \multirow{2}{*}{0} & $\mathrm{n}$ & 10 & 24 & 15 & 9 & 9 & 9 \\
\hline & & $\%$ & 100 & 82,8 & 88,2 & 100 & 50 & 100 \\
\hline & \multirow{2}{*}{1} & $\mathrm{n}$ & 0 & 4 & 2 & 0 & 9 & 0 \\
\hline & & $\%$ & 0 & 13,8 & 11,8 & 0 & 50 & 0 \\
\hline & \multirow{2}{*}{2} & $\mathrm{n}$ & 0 & 1 & 0 & 0 & 0 & 0 \\
\hline & & $\%$ & 0 & 3,4 & 0 & 0 & 0 & 0 \\
\hline & \multirow[t]{2}{*}{3} & $\mathrm{n}$ & 0 & 0 & 0 & 0 & 0 & 0 \\
\hline & & $\%$ & 0 & 0 & 0 & 0 & 0 & 0 \\
\hline \multicolumn{3}{|c|}{$\begin{array}{c}\text { Valores } \mathrm{p} \text { (MON2 vs. } \\
\text { PAT2, borde) }\end{array}$} & - & 0,00220 & 0,01819 & 0,38889 & 0,00133 & 0,1246 \\
\hline \multicolumn{3}{|c|}{$\begin{array}{c}\text { Valores } \mathrm{p}(\mathrm{MON} 2 \text { vs. } \\
\text { PAT2, superficie) }\end{array}$} & - & 0,30527 & 0,32126 & 1 & 0,01180 & - \\
\hline
\end{tabular}

Tabla 3. Prevalencias ( $n$ y \%) de lesiones articulares en borde y superficie en MON2 y PAT2, considerando grados de severidad. Se incluyen valores $\mathrm{p}$ del test Mann-Whitney (en gris se resaltan las diferencias significativas). variable que más contribuyó a la diferenciación (Figura 2, Tabla S7). En la comparación de los miembros inferiores de MON1-PAT1, dentro del Componente 1 (62,6\% de la varianza explicada), fue la formación ósea en la zona 1 (FO1) la variable que más contribuyó (Figura 2, Tabla S7). De acuerdo con estas variables, en MON1, las entesis más afectadas en los miembros superiores fueron el bíceps, el braquial y el subescapular (Tabla S8). El iliopsoas y el plantar fueron las entesis más afectadas en miembros inferiores. En PAT1, las entesis más afectadas fueron el bíceps, el flexor, el braquial y el extensor en los miembros superiores. Las inserciones del iliopsoas, glúteo menor y medio y el plantar fueron las más afectadas en miembros inferiores. Diferencias significativas entre desiertos solo se observaron en el bíceps $(p=0,0023)$ y el flexor $(p=0,00368)$. Se destaca un gran solapamiento entre los conjuntos MON1 y PAT1, observado en el ACP, que muestra que la segregación es mayor a

\begin{tabular}{|c|c|c|c|c|c|c|c|c|c|c|}
\hline & \multicolumn{2}{|c|}{ MON1 } & \multicolumn{2}{|c|}{ PAT1 } & \multicolumn{2}{|c|}{ MON2 } & \multicolumn{2}{|c|}{ PAT2 } & \multirow{2}{*}{$\begin{array}{c}\text { Valores p } \\
\text { (MON1 vs. } \\
\text { PAT1) }\end{array}$} & \multirow{2}{*}{$\begin{array}{c}\text { Valores p } \\
\text { (MON2 vs } \\
\text { PAT2) }\end{array}$} \\
\hline & $\mathbf{n}$ & $\%$ & $\mathbf{n}$ & $\%$ & $\mathbf{n}$ & $\%$ & $\mathbf{n}$ & $\%$ & & \\
\hline Superiores/BOR & 91 & 29,6 & 12 & 34,3 & 3 & 12 & 32 & 57,1 & 0,69983 & 0,00014 \\
\hline Inferiores/BOR & 41 & 26,6 & 8 & 23,5 & 2 & 11,1 & 23 & 62,2 & 0,75958 & 0,00039 \\
\hline Superiores/SUP & 29 & 9 & 0 & 0 & 1 & 4 & 7 & 12,5 & 0,06563 & 0,23942 \\
\hline Inferiores/SUP & 5 & 3,2 & 3 & 8,6 & 0 & 0 & 9 & 25 & 0,16042 & 0,02214 \\
\hline
\end{tabular}

Tabla 4. Prevalencias ( $n$ y \%) de lesiones articulares en borde y superficie por porción anatómica (miembros superiores e inferiores), sin discriminar por grados de severidad. Se incluyen valores $p$ del test Mann-Whitney (en gris se resaltan las diferencias significativas). 
nivel intraconjunto (dentro de cada desierto) que entre conjuntos.

En la comparación de los miembros superiores del segundo bloque temporal (MON2-PAT2), el Componente 1 explicó el 47,7\% de la varianza (Figura 2, Tabla S7). Dentro de este componente, la variable de mayor peso en la diferenciación fue FO1. En la comparación de los miembros inferiores de MON2-PAT2, el Componente 1 explicó el 58,1\% de la varianza, y la variable que más contribuyó dentro de dicho componente fue nuevamente FO1 (Figura 2, Tabla S7). En MON2, las entesis afectadas de miembros superiores fueron el braquial, el extensor, el tríceps y el bíceps (Tabla S9). En los miembros inferiores, el glúteo menor, el glúteo medio y el iliopsoas mostraron las prevalencias más altas de cambios. En PAT2, los porcentajes fueron más altos que con respecto a MON2. Las inserciones del braquial, del supraespinoso y del subescapular, del iliopsoas y los plantares fueron las más afectadas. No se encontraron diferencias significativas entre desiertos.

\section{Miembros superiores e inferiores}

Para ambos tipos de entesis se destacan las mayores prevalencias de cambios asociados con robusticidad y formación ósea en zona 1, en los miembros inferiores y superiores de las muestras de Patagonia en relación con las de Monte para ambos períodos (Figura 3). Considerando las prevalencias de robusticidad entre desiertos y por porción anatómica, se observaron diferencias significativas para el período 2000-1000 años AP solo a nivel de los miembros inferiores $(p=0,02287)$, no así entre los superiores $(p=0,44541)$. En el período 1000-200 años AP se observaron diferencias significativas a nivel de miembros superiores $(p=0,00098)$ e inferiores $(p=0,00049)$. Teniendo en consideración las prevalencias de formación ósea en zona 1 en el período 2000-1000 años AP, no se observaron diferencias significativas entre los desiertos (entre miembros superiores, $p=0,23399$; entre miembros inferiores, $p=0,6062$ ). En el período 1000-200 años $\mathrm{AP}$, tampoco se observaron diferencias significativas entre las porciones anatómicas (entre miembros superiores, $p=0,05272$; entre miembros inferiores, $p=0,05653)$.

\section{DISCUSIÓN Y CONCLUSIONES}

De acuerdo con las hipótesis y expectativas planteadas al inicio, se esperaba que los miembros inferiores de los individuos del Monte presentaran

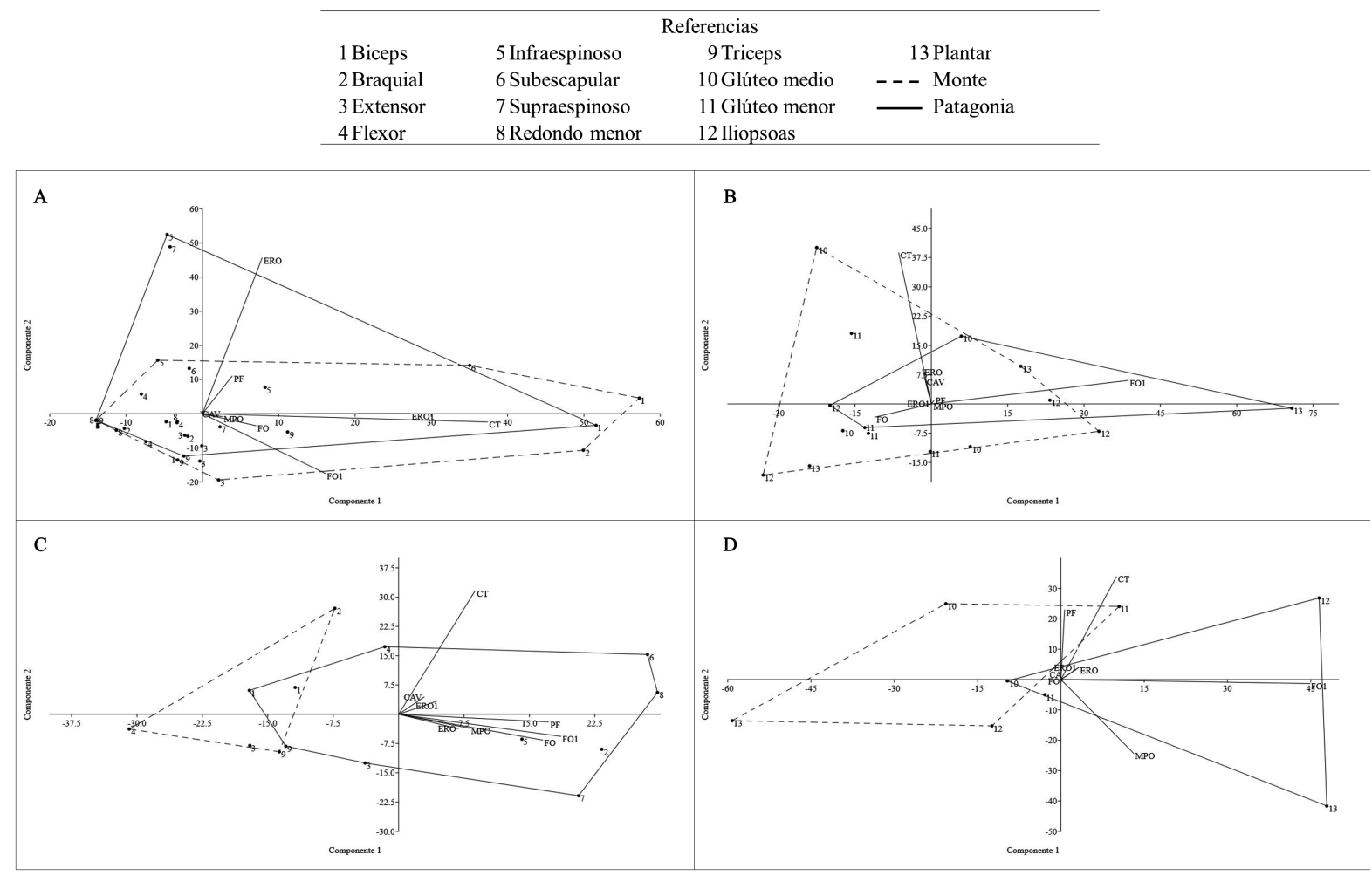

Figura 2. ACP que compara los cambios en entesis fibrocartilaginosas entre los conjuntos MON1-PAT1 y MON2-PAT2 por porción anatómica. A. Miembros superiores de los conjuntos MON1-PAT1. B. Miembros inferiores de los conjuntos MON1PAT1. C. Miembros superiores de los conjuntos MON2-PAT2. D. Miembros inferiores de los conjuntos MON2-PAT2. 


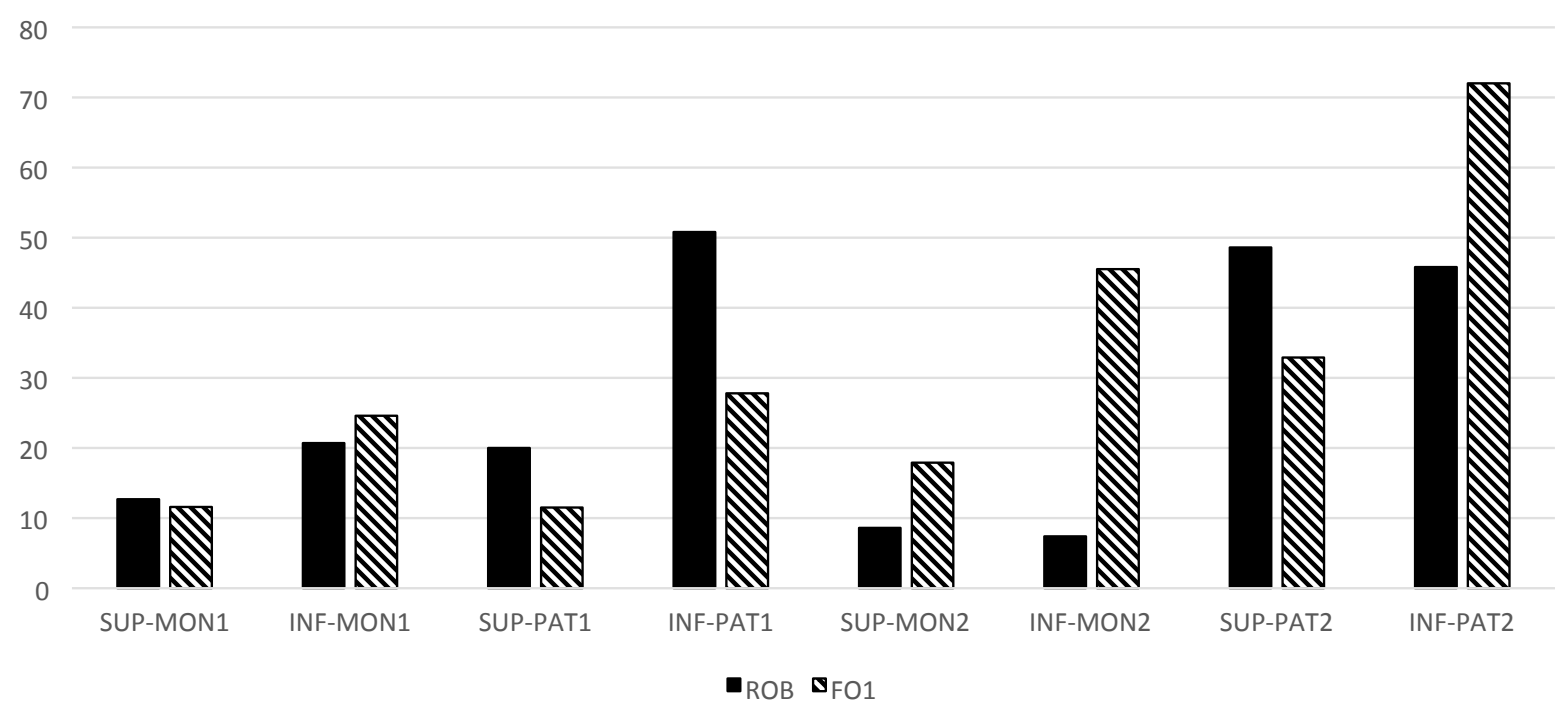

Figura 3. Porcentajes de cambios entésicos por porción anatómica (miembros superiores e inferiores), considerando robusticidad (ROB) y formación ósea en zona 1 (FO1) en los cuatro conjuntos (MON1, PAT1, MON2, PAT2).

bajas prevalencias de cambios entésicos y de lesiones articulares respecto de las tendencias identificadas en Patagonia para la misma porción anatómica. Mayores prevalencias de LA y CE se esperaban en los miembros superiores de Monte con respecto a los de Patagonia. Se propuso además la continuidad de estas tendencias, en la medida en que la agricultura se iniciaría en Monte y la caza-recolección continuaría en Patagonia.

Para el primer bloque temporal, inmediatamente posterior al ingreso de los cultígenos en el valle del Atuel, los resultados no permitieron sostener la hipótesis unánimemente. En términos de LA, contrariamente a lo esperado, se observó que estuvieron más afectados los miembros inferiores en la muestra de Monte que en la de Patagonia; aunque la diferencia no es significativa. Las entesis fibrosas y fibrocartilaginosas de miembros inferiores se mostraron menos afectadas en Monte que en Patagonia, aunque solo de manera significativa en el caso de las fibrosas. En los miembros superiores, contrariamente a lo esperado, los porcentajes de afección fueron más bajos en la muestra de Monte que en la de Patagonia, tanto a nivel de las lesiones articulares como de los cambios en entesis fibrosas y fibrocartilaginosas, aunque solo de manera significativa en el caso de las fibrocartilaginosas. Considerando estos resultados, una interpretación posible es que en Monte los individuos habrían implementado estrategias tendientes a la reducción de la movilidad, mientras que en Patagonia la movilidad pudo haber sido más alta. Por otra parte, es factible sugerir que las actividades manuales habrían sido diferentes entre desiertos, teniendo en cuenta las mayores prevalencias observadas en los miembros superiores del conjunto de Patagonia, como también las diferencias en las entesis afectadas en dicha porción anatómica.

No se descarta que lo observado a nivel de los miembros inferiores también pueda ser el resultado de diferencias en la manera en que se pautan las actividades cotidianas (i.e., posturas, cantidad y duración de las tareas, mayor o menor resistencia al estrés mecánico, etc.), no necesariamente ligadas a la movilidad. Asimismo, las tendencias podrían explicarse también por diferencias en la composición de las muestras de ambos conjuntos en cuanto al sexo y la edad de los individuos. De acuerdo con esto, los individuos de mayor edad y/o de sexo masculino presentarían prevalencias más altas de CE y LA que los más jóvenes y/o de sexo femenino. Si bien no fue posible controlar estas variables en esta instancia de investigación -dadas las características de los entierros-, los datos generales de las muestras indican para ambos conjuntos una prevalencia alta de individuos maduros de ambos sexos, por lo que estarían equilibrados desde un punto de vista comparativo.

Las tendencias sugeridas en cuanto a la movilidad adquieren relevancia en relación con la presencia de maíz en la dieta de algunos grupos de MON1 y PAT1 (Gil et al., 2017; Peralta, 2019). Mientras que en Monte la presencia del maíz pudo generar un impacto a nivel organizativo -lo que redefiniría las estrategias de movilidad y el aprovisionamiento de recursos-, en Patagonia, las estrategias pudieron ser más conservadoras, con un estilo de vida más 
móvil y más dependiente de la caza-recolección, aunque el maíz pudiera haber estado presente en las dietas. En este contexto, el hecho de que las prevalencias más bajas en los miembros inferiores de Monte no hayan sido significativas para todos los indicadores considerados podría responder a una fase exploratoria en el consumo de recursos domesticados, con un impacto todavía leve en las actividades cotidianas.

En cuanto a las actividades manuales, la mayor prevalencia de cambios en los miembros superiores de Patagonia en relación con los de Monte puede explicarse como el resultado de la realización de actividades vinculadas a la caza-recolección con mayor frecuencia (e.g., uso de arco y flecha), sumadas a un uso más intensivo de elementos de procesamiento (e.g., molienda) (Molleson, 1994; Eshed et al., 2004; Marchi et al., 2006; Larsen y Ruff, 2011). La intensificación de la molienda en Patagonia se ve apoyada por trabajos recientes en isótopos estables, en los que se observa un consumo importante de recursos vegetales, tanto silvestres como domésticos (Peralta, 2019). La realización de actividades manuales distintivas entre los individuos de Monte y Patagonia se sostiene también por las diferencias significativas en las entesis que se encuentran más afectadas en uno y otro ambiente.

Para el segundo bloque temporal, los resultados obtenidos apoyan parcialmente la hipótesis, al indicar que en Monte los miembros inferiores estuvieron significativamente menos afectados que en Patagonia, en cuanto a las lesiones articulares y los cambios en entesis fibrosas. Los miembros superiores, contrariamente a lo esperado, no se mostraron más afectados en la muestra de Monte con respecto a la de Patagonia. Los isótopos estables en MON2 y PAT2 mostraron que el consumo de maíz solo se habría sostenido entre individuos de MON2 (Gil et al., 2017; Peralta, 2019), en coincidencia con lo observado a partir de los indicadores de uso del cuerpo. En cuanto a las altas prevalencias observadas en la muestra de PAT2, una explicación posible tiene que ver con la mayor frecuencia de individuos maduros en este conjunto. Otra explicación deriva de la procedencia de algunos de los individuos que integran la muestra. Corresponden al sitio CME, fechado en $341 \pm 37$ años AP, cuyas ocupaciones se han atribuido a grupos mapuche en contacto con europeos (Peralta, 2019). Las nuevas condiciones de vida, entre las que se incluye el uso del caballo, pudieron impactar fuertemente en las actividades físicas y en los indicadores óseos resultantes.

Las características del ambiente, condicionadas en gran medida por la presencia de la diagonal árida y el régimen de precipitaciones, no habrían favorecido una alta dependencia en la agricultura en ninguno de los desiertos que motivara una reducción drástica de la movilidad o un cambio sustancial en las actividades físicas, aunque los cultígenos -obtenidos mediante diferentes vías- podrían haber constituido un complemento alimentario para algunos grupos humanos. El hecho de que se trate de un área transicional implica que las estrategias adoptadas deben ser igualmente diversas y versátiles, susceptibles de variar en tiempo y espacio.

Parte de los patrones aquí observados pueden responder efectivamente a la realización de actividades diferentes y cambios en la movilidad, como también pueden explicarse por otros factores, como la edad, que no fue posible controlar, dadas las características de la muestra. También será necesaria la profundización de estos estudios incorporando estimaciones de sexo y controlando la relación con el tamaño corporal para continuar con la discusión acerca de los patrones de uso del cuerpo en un área en la que coexistieron modos de subsistencia diversos.

\section{Agradecimientos}

Este trabajo ha sido posible gracias a los Dres. Adolfo F. Gil y Leandro H. Luna, y al apoyo institucional de IDEVEA-CONICET, Universidad Tecnológica Nacional-Facultad Regional San Rafael y Museo de Historia Natural de San Rafael. Gracias a Vivi Seitz por su mapa y su maravillosa amistad.

\section{REFERENCIAS CITADAS}

Aufderheide, A. y Rodriguez-Martín, G. (1998). The Cambridge encyclopedia of human pathology. Cambridge University Press.

Benjamin, M., Evans, E. J. y Copp, L. (1986). The histology of tendon attachments to bone in man. Journal of Anatomy, 149, 89-100.

Benjamin, M., Kumai, T., Milz, S., Boszczyk, B. M., Boszczyk, A. A. y Ralphs, J. R. (2002) The skeletal attachment of tendons-tendon 'entheses'. Comparative Biochemistry and Physiology - Part A: Molecular \& Integrative Physiology, 133(4), 931-45. 
Benjamin, M. y Ralphs, J. R. (1998) Fibrocartilage in tendon and ligaments- an adaptation to compressive load. Journal of Anatomy, 193(4): 481-494.

Berenbaum, F. (2013) Osteoarthritis as an inflammatory disease (osteoarthritis is not osteoarthrosis!). Osteoarthritis and Cartilage, 21, 16-21.

Bridges, P. (1989). Changes in activity with the shift to agriculture in the southeastern United States. Current Anthropology, 30, 385-394.

Bridges, P. (1992). Prehistoric arthritis in the Americas. Annual Review of Anthropology, 21, 67-91.

Bridges, P. (1994). Vertebral arthritis and physical activities in the prehistoric Southeastern United States. American Journal of Physical Anthropology, 93, 83-93.

Eshed, V., Gopher, A., Galili, A. y Hershkovitz, I. (2004). Musculoskeletal stress markers in Natufian hunter gatherers and Neolithic farmers in the Levant: The upper limb. American Journal of Physical Anthropology, 123, 303-315.

Giannotti, P. S. (2016). Marcadores de estrés ocupacional en poblaciones históricas del norte de Mendoza (s. XVI-XVII): primeros resultados exploratorios. Comechingonia, 20(1), 81-110.

Gil, A. (2003). Zea mays on the South American Periphery: Chronology and Dietary Importance. Current Anthropology, 44(2), 295-300.

Gil, A. y Neme, G. (Eds.) (2010). Registro arqueológico en la cuenca media del Atuel: viejos y nuevos problemas; viejos y nuevos datos. En Condiciones paleoambientales y ocupaciones humanas durante la transición Pleistoceno-Holoceno y Holoceno de Mendoza (pp. 239-275). Sociedad Argentina de Antropología.

Gil, A., Menéndez, L., Atencio, J., Peralta, E., Neme, G. y Ugan, A. (2017). Estrategias humanas, estabilidad y cambio en la frontera Agrícola sur americana. Latin American Antiquity, 29(1), 6-26.

Gil, A., Tykot, R., Neme, G. y Shelnut, N. (2006). Maize on the frontier: isotopic and macrobotanical data from central-western Argentina. En J. Staller, R. H. Tykot y B. Benz (Eds.), Histories of Maize (pp. 199-214). Elsevier Academic Press.

Hammer, O., Harper, D. y Ryan, P. (2001). PAST: Paleontological Statistics Software Package for Education and Data Analysis. Paleontología Electrónica, 4(1), 1-9.

Hawkey, D. y Merbs, C. (1995). Activity-Induced Musculoskeletal Stress Markers (MSM) and Subsistence Strategy Changes among Hudson Bay Eskimos. International Journal of Osteoarchaeology, 5, 324-338.
Henderson, C. (2008). When hard work is disease: the interpretation of enthesopathies. En M. Brickley y M. Smith (Eds.), Proceedings of the $8^{\text {th }}$ annual conference of the British Association of Biological Anthropology and Osteoarchaeology (pp. 17-25). Archaeopress.

Henderson, C. y Alves-Cardoso, F. (2013). Special issue entheseal changes and occupation: technical and theoretical advances and their applications. International Journal of Osteoarchaeology, 23, 127-134.

Henderson, C., Mariotti, V., Pany-Kucera, D., Villotte, S. y Wilczak, C. (2013). Recording specific features of fibrocartilaginous entheses: preliminary results of the Coimbra standard method. International Journal of Osteoarchaeology, 23, 152-162.

Henderson, C., Mariotti, V., Pany-Kucera, D., Villotte, S. y Wilczak, C. (2016a). The New "Coimbra Method": A Biologically Appropriate Method for Recording Specific Features of Fibrocartilaginous Entheseal Changes. International Journal of Osteoarchaeology, 26, 925-932.

Henderson, C., Wilczak, C. y Mariotti, V. (2016b). Commentary: an update to the new Coimbra method for recording entheseal changes. International Journal of Osteoarchaeology, 27(3), 521-522.

Henderson, C., Mariotti, V., Santos, F., Villotte, S. y Wilczak, C. A. (2017). The new Coimbra method for recording entheseal changes and the effect of age-at-death. BMSAP, 29, 140-149. doi.org/10.1007/ s13219-017-0185-x

Henderson, C., Salega, S. y Silva, A. M. (2018). Portuguese women's activity in the past: comparing entheseal changes through time. Annales Universitatis Apulensis. Series Historica, 22(1), 195-222.

Holt, B. (2003). Mobility in upper Paleolithic and Mesolithic Europe: evidence from the lower limb. American Journal of Physical Anthropology, 122, 200-215.

Holt, B. y Formicola, V. (2008). Hunters of the Ice Age: the biology of Upper Paleolithic people. Yearbook of Physical Anthropology, 51, 70-99.

Jurmain, R. (1977). Stress and Etiology of Osteoarthritis. American Journal of Physical Anthropology, 46, 353-366.

Jurmain, R. (1980). The Pattern of Involvement of Appendicular Degenerative Joint Disease. American Journal of Physical Anthropology, 53, 143-150.

Jurmain, R. y Villotte, S. (2010). Terminology. Entheses in medical literature and physical anthropology: a brief review. Coimbra. Document published online in 4th February following the Workshop in Musculoskeletal Stress Markers (MSM): limitations and achievements in the reconstruction of past activity patterns, University 
of Coimbra, July 2-3, 2009. Coimbra, CIAS - Centro de Investigação em Antropologia e Saúde. http://www. uc.pt/en/cia/msm/MSM_terminology3

Jurmain, R., Alves-Cardoso, F., Henderson, C. y Villotte, S. (2012). Bioarchaeology's Holy Grail. The Reconstruction of Activity. En A. Grauer (Ed.), A Companion to Paleopathology (pp. 531-552). Wiley-Blackwell.

Lagier, R. (2006). Bone eburnation in rheumatic diseases: a guiding trace in today's radiological diagnosis and in paleopathology. Clinical Rheumatology, 25(12), 7-131.

Larsen, C. (1984). Health and disease in prehistoric Georgia: the transition to agriculture. En M. N. Cohen y G. J. Armelagos (Eds.), Paleopathology at the origins of agriculture (pp. 367-392). Academic Press.

Larsen, C. y Ruff, C. (2011). "An External Agency of Considerable Importance": The Stresses of Agriculture in the Foraging-to-Farming Transition in Eastern North America. En R. Pinhasi y J. Stock (Eds.), Human Bioarchaeology of the Transition to Agriculture (pp. 293-325). Wiley-Blackwell.

Luna, L., Aranda, C. y Amorim-Alves, A. (2017). Reflexiones sobre el relevamiento y análisis comparativo de patologías osteoarticulares en restos esqueletales humanos. Revista Argentina de Antropología Biológica, 19(1), 7-14.

Manuila, L., Manuila, A., Lewalle, P. y Nicoulin, M. (2000). Dicionário médico. Climepsi.

Marchi, D., Sparacello, V., Holt, B. y Formicola, V. (2006). Biomechanical approach to the reconstruction of activity patterns in Neolithic Western Liguria, Italy. American Journal of Physical Anthropology, 131, 447-455.

Marchi, D., Sparacello, V. y Shaw, C. (2011). Mobility and lower limb robusticity of a pastoralist neolithic population from North-Western Italy. En R. Pinhasi y J. Stock (Eds.), Human Bioarchaeology of the Transition to Agriculture (pp. 317-346). Wiley-Blackwell.

Molleson, T. (1994). The Eloquent bones of Abu Hureya. Scientific American, 271(2), 70-75.

Neme, G. (2007). Cazadores-recolectores de altura en los Andes meridionales: el alto valle del río Atuel. BAR International Series 1591. Archaeopress.

Neme, G. y Gil, A. (2012). El registro arqueológico del sur de Mendoza en perspectiva biogeográfica. En A. Gil y G. Neme (Eds.), Paleoecología humana en el sur de Mendoza: perspectivas arqueológicas (pp. 255-279). Sociedad Argentina de Antropología.

Neumann, D. (1999). Joint deformity and dysfunction: a basic review of underlying mechanism. Arthritis Care and Research, 12(2), 139-151.
Novellino, P. y Neme, G. (1999). Hallazgo de restos humanos en Laguna Blanca y El Sosneado (Sur de Mendoza). Actas del XII Congreso Nacional de Arqueología Argentina, Tomo 3 (pp. 256-260). La Plata.

Nolte, M. y Wilczak, C. (2013). Three-dimensional Surface Area of the Distal Biceps Enthesis, Relationship to Body Size, Sex, Age and Secular Changes in a 20th Century American Sample. International Journal of Osteoarchaeology, 23, 163-174.

Peralta, E. (2017). Cambios y continuidades en la movilidad humana a finales del Holoceno tardío: cambios entésicos, lesiones articulares e isótopos estables en el sur de Mendoza. Revista del Museo de Antropología, 10(2), 157-166.

Peralta, E. (2019). Demografía humana, dieta y actividad en los límites de la dispersión agrícola: tendencias bioarqueológicas en el sur de Mendoza a finales del holoceno tardío [Tesis doctoral inédita, Universidad de Buenos Aires].

Rogers, J. y Waldron, T. (1995). A field guide to joint disease in archaeology. John Wiley \& Sons.

Rogers, J., Shepstone, L., Dieppe, P. (2004). Is osteoarthritis a systemic disorder of bone? Arthritis \& Rheumatism, 50(2), 452-457.

Roosevelt, A. (1984). Population, health, and the evolution of subsistence: conclusions from the conference. En M. N Cohen y G. J. Armelagos (Eds.), Paleopathology at the origins of agriculture (pp. 559-583). Academic Press.

Rothschild, B. (1997). Porosity: a curiosity without diagnostic significance. American Journal of Physical Anthropology, 104, 529-533.

Salega, S. (2016). Prácticas cotidianas, niveles de actividad física y modos de vida en poblaciones del sector austral de las Sierras Pampeanas durante el Holoceno tardío [Tesis doctoral inédita, Universidad Nacional de Córdoba].

Santos, A. L., Alves-Cardoso, F., Assis, S. y Villotte, S. (2011). The Coimbra Workshop in Musculoskeletal Stress Markers (MSM): an annotated review. Antropología Portuguesa, 28, 135-161.

Scabuzzo, C. (2010). Actividad, Patología y Nutrición de los Cazadores Recolectores Pampeanos [Tesis doctoral inédita, Universidad Nacional de La Plata].

Scabuzzo, C. (2012). Estudios bioarqueológicos de Marcadores de Estrés Ocupacional en cazadores recolectores pampeanos del Holoceno tempranomedio. Análisis de la serie esqueletal de Arroyo Seco 2. Revista Argentina de Antropología Biológica, 14(1), 17-31. 
Schrader, S. (2012). Activity Patterns in New Kingdom Nubia: An Examination of Entheseal Remodeling and Osteoarthritis at Tombos. American Journal of Physical Anthropology, 149(1), 60-70.

Seibel, M., Robins, S., Bilezikian, J. (eds.) (2006). Dynamics of bone and cartilage metabolism. Elsevier.

Solomon, L. (2001). Clinical features of osteoarthritis. En S. Ruddy, E. Harris, C. Sledge (Eds.), Kelley's Textbook of Rheumatology (pp. 1409-1418). Saunders.

Sparacello, V., Samsel, M., Villotte, S., Varalli, A., Schimmenti, V. y Sineo, L. (2020). Inferences on Sicilian Mesolithic subsistence patterns from cross-sectional geometry and entheseal changes. Archaeological and Anthropological Sciences, 12, 101. doi.org/10.1007/s12520-020-01044-y

Stirland, A. (1998). Musculoskeletal evidence for activity: Problems of evaluation. International Journal of Osteoarchaeology, 8, 354-362.

Stock, J., O'Neill, M., Ruff, C., Zabecki, M., Shackelford, L. y Rose, J. (2011). Body Size, skeletal biomechanics, mobility and habitual activity from the Late Palaeolithic to the Mid-Dynastic Nile Valley. En R. Pinhasi y J. Stock (Eds.), Human Bioarchaeology of the Transition to Agriculture (pp. 347-367). Wiley-Blackwell.

Varalli, A., Villotte, S., Dori, I., Sparacello, V. (2020). New insights into activity-related functional adaptations and entheseal changes in Neolithic Liguria (northwestern Italy). BMSAP, 32(1-2), 34-58. doi.org/10.3166/ bmsap-2020-0072

Villotte, S. y Knüsel, C. (2013). Understanding Entheseal Changes: Definition and Life Course Changes. International Journal of Osteoarchaeology, 23, 135-146.

Villotte, S., Castex, D., Couallier, V., Dutour, O., Knüsel, C. J., Henry-Gambier, D. (2010). Enthesopathies as occupational stress markers: evidence from the upper limb. American Journal of Physical Anthropology, 142(2), 224-234.

Waldron, T. (2009). Paleopathology. Cambridge University Press.

Wolff, J. (1892). Das Gesetz der Transformation der Knochen. A. Hirchwild. 